\title{
$\mathrm{icm}^{\circ}$
}

\section{Some examples in the theory of Borel sets \\ by}

\section{Stephen Willard (Edmonton)}

All spaces are normal, unless the contrary is explicitly stated, and "normal" implies. "Hausdorff". Our notation and terminology about Borel sets follow [2]. In particular, $\alpha, \beta$ and $\gamma$ are always ordinals less than the first uncountable ordinal. The real line with its usual topology is denoted $\boldsymbol{R}$ and the subspace of $\boldsymbol{R}$ consisting of the irrationals is denoted $\boldsymbol{P}$.

In perfectly normal spaces, Borel sets are particularly easy to handle because they can be resolved into an increasing transfinite sequence of classes: $G_{0}, G_{1}, \ldots, G_{\alpha}, \ldots$ The important property of perfectly normal spaces here is that every closed set in such a space is a $G_{\delta}$. Less is needed: it is enough that every closed set be a $G_{\alpha}$ for some $\alpha$. In Section 1 , for each fixed $\alpha$, we construct a space such that $\alpha$ is the smallest ordinal for which every closed set in the space is a $G_{a}$, answering a correspondence question of A. H. Stone. As an easy corollary, the disjoint union of the spaces thus constructed has the property that every open set is Souslin (generated from the closed sets by operation A), but in which there is an open set which is not an $F_{\alpha}$ for any $\alpha$.

The problem of producing a space in which each closed set is a $G_{*}$ for some $\alpha$ (depending on the closed set), but in which.no $\alpha$ works for all closed sets, is badgered in Section 2. The resulting counterexample is Hausdorff, but fails to be normal and depends on the continuum hypothesis for its existence. It would be interesting to have a normal example.

In perfectly normal spaces (see [2], pp. 347-348), the family of Borel sets coincides with the smallest family containing the open sets and closed under countable intersection and countable disjoint union, and the same is true if the closed sets are used in place of the open sets. In Section 3 an example (one of those used in Section 1) is given to show that perfect normality is not needed for either of these assertions. Previous examples of a similar nature have been given by Frolik ([1], p. 166).

1. For each $\alpha, 1<\alpha<\omega_{1}$, we will provide a (normal) space $X_{\alpha}$ in which $\alpha$ is the smallest ordinal such that every closed set is a $G_{\alpha}$. As a set, 
$X_{\alpha}$ will be the real line. Let $A_{\alpha}$ be a subset of the line which, in the usual topology, is a non-trivial $G_{a}$ set (i. e., a $G_{a}$ set but not a $G_{\beta}$ set for any $\beta<\alpha-$ such sets exist in the line, see [2], p. 371). The open sets in $X_{\alpha}$ are now defined to be the sets of the form $U \cup V$, where $U$ is open in the usual line topology and $V$ is any subset of $X_{\alpha}-A_{\alpha}$. [For $\alpha=2, A_{\alpha}$ could be the rationals. The resulting space $X_{2}$ was first used by Michael [3]] ( ${ }^{1}$ ). As Michael has pointed out, any space constructed in this way (by "discretizing" a set, in our case $X_{a}-A_{\alpha}$ ) from a metric space is hereditarily paracompact and thus normal.

Now note that $A_{\alpha}$ is a $G_{a}$ in $X_{a}$ and has for its relative topology in $X_{\alpha}$ its usual metric topology. Then if $F$ is closed in $X_{\alpha}, F=\left[F \cap\left(X-A_{\alpha}\right)\right] \cup$ $\cup\left(F \cap A_{a}\right)$, the former being open in $X_{a}$ and the latter being a $G_{\delta}$ set in $A_{\alpha}$ (since it is a closed subset of the metric space $A_{\alpha}$ ) and thus a $G_{\alpha}$ in $X_{\alpha}$. It follows that every closed set $F$ is a $G_{a}$ in $X_{\alpha}$.

Next, if $Q$ is an open set in $X_{\alpha}$, it is easily seen that an open set $P$ in the usual line topology exists for which $P \cap A_{\alpha}=Q \cap A_{\alpha}$ and $P \subset Q$. It follows that if $A_{\alpha}$ is a $G_{\beta}$ in $X_{\alpha}$, it is a $G_{\beta}$ in the line, so this cannot happen for $\beta<\alpha$.

Now if $X$ is the disjoint union of the spaces $\dot{X}_{\alpha}$ just constructed; the open set $U\left(X_{\alpha}-A_{\alpha}\right)$ in $X$ is not an $F_{\alpha}$ for any $\alpha$. But every open set in $X$ is the union of $\kappa_{1} F_{a}$ sets (for variable $a$ ) and thus is a Souslin set.

This fulfills the promises made for Section 1 in the introduction.

2. The program here is to provide an example showing every closed set in a space can be represented as a $G_{\alpha}$ without it being possible to fix $\alpha$. Some preliminary lemmas are needed; unfortunately, even with these, the resulting example has the lack of common decency to be non-normal.

LEMMA. For each $\alpha, 1 \leqslant \alpha<\omega_{1}$, a non-trivial $G_{\alpha}$ set $P_{a}$ can be found in $\boldsymbol{R}$ such that

(1) $P_{a} \cap P_{\beta}=\emptyset$, if $\alpha \neq \beta$,

(2) $P_{1} \cup \ldots \cup P_{a}$ is not a $G_{\beta}$ for $\beta<\alpha$,

(3) $\bigcup_{1 \leqslant a<\infty_{1}} P_{\alpha}=\boldsymbol{R}$ [continuum hypothesis].

Proof. For each $r \in P$, let $P(r)=\{(q, r) \mid q \in P\} \subset P \times P$. Assign each ordinal $\alpha, 1 \leqslant \alpha<\omega_{1}$, to some $P(r)$, hereafter called $P\left(r_{a}\right)$. From [2], a non-trivial $G_{\alpha}$ subset $P_{\alpha}^{\prime}$ of $P\left(r_{a}\right)$ exists, and $P_{a}^{\prime}$ will still be a nontrivial $G_{a}$ in $P \times P$. But $P \times P$ is homeomorphic to $P$ (proof: the irrationals are a countable product of copies of the integers), say $h$ is a homeomorphism of $P \times P$ onto $P$. Let $P_{\alpha}^{*}=h\left(P_{\alpha}^{\prime}\right)$. Now $P_{\alpha}^{*}$ is a non-trivial $G_{\alpha}$

(1) And I acknowledge a debt to J. R. Isbell and A. H. Stone, each of whom pointed out the similarity of my (then different) example for $\alpha=2$ to Michael's example. set in $P$ and hence in $\boldsymbol{R}$, and the $P_{a}^{*}$ are mutually disjoint. Moreover, if $P_{1}^{*} \cup \ldots \cup P_{a}^{*}$ is a $G_{\beta}$, then $P_{1}^{\prime} \cup \ldots \cup P_{\alpha}^{\prime}$ is a $G_{\beta}$, and hence $P_{\alpha}^{\prime}=\left(P_{1}^{\prime} \cup\right.$ $\left.\cup \ldots \cup P_{a}^{\prime}\right) \cap P\left(r_{a}\right)$ is a $G_{\beta} \cap G_{\delta}$, hence a $G_{\delta}$, if $\beta \geqslant 1$. Thus this cannot happen for $\beta<\alpha$.

We have the properties of the theorem except for 3. To get this, assume the continuum hypothesis and write the real numbers as a transfinite sequence $x_{1}, x_{2}, \ldots, x_{\alpha}, \ldots, \alpha<\omega_{1}$. Let $P_{\alpha}^{+}=P_{a}^{*} \cup\left\{x_{\alpha}\right\}$. Then $P_{\alpha}^{+}$is still a non-trivial $G_{\alpha}$ and $P_{3}^{+} \cup \ldots \cup P_{\alpha}^{+}$differs from $P_{1}^{*} \cup \ldots \cup P_{\alpha}^{*}$ by a countable set $\left(\therefore\right.$ an $\left.F_{\sigma}\right)$ and hence cannot be a $G_{\beta}$. The $P_{\alpha}^{+}$are not mutually disjoint, but if we now set $P_{\alpha}=P_{\alpha}^{+}-\left\{x_{\beta} \mid \beta<\alpha\right\}$, we obtain mutually disjoint sets $P_{\alpha}$ having all the properties required (by arguments similar to those already given).

We proceed to the construction of the counterexample of this section. For each $\alpha, 1 \leqslant \alpha<\omega_{1}$, let $P_{\alpha}$ be a non-trivial $G_{a}$ subset of $\boldsymbol{R}$ having properties 1 through 3 of the Lemma above. We will retopologize the line, now called $X$, as follows: the neighborhoods of a point $p$ in $P_{\alpha}$ will have the form $U-\left(\bigcup_{\beta} P_{\beta}\right)$ where $U$ is a usual linear neighborhood of $p$. It is clear, then, that every set open in the usual topology is open in this topology.

Setting $B_{a}=P_{1} \cup \ldots \cup P_{\alpha}$, it is clear that $B_{\alpha}$ is closed and a $G_{\alpha}$ in $X$. But it is not a $G_{\beta}$ for $\beta<\alpha$, for if $G$ is any open subset of $X$ meeting $B_{\alpha}$, each point $p$ of $G \cap B_{\alpha}$ has a neighborhood $U_{p}-\left(\bigcup_{\beta} P_{\beta}\right)$, where $U$ is a linear neighborhood of $p$ and $\gamma \leqslant \alpha$, contained in $G$. Let $H=\bigcup_{p \in G \cap B_{\alpha}} U_{p}$. Then, easily, if $B_{\alpha}$ is generated as a $G_{\beta}$ in $X$ by $G_{1}, G_{2}, \ldots$, it will be generated as a $G_{\beta}$ in $\boldsymbol{R}$ by $H_{1}, H_{2}, \ldots$, and this is not possible, by Part 2 of the lemma, for $\beta<\alpha$.

Thus closed non-trivial $G_{a}$ sets exist in $X$ for arbitrarily high elass $\alpha<\omega_{1}$. It remains to show that each closed set in $X$ is a $G_{\alpha}$ for some $\alpha$. We attack the open sets, showing each to be an $F_{\alpha}$ for large enough $\alpha$.

Let $W$ be open in $X$. For $p \in W \cap P_{\alpha}$, let $\nabla_{p}=U_{p}-\bigcup_{\beta<\alpha} P_{\beta}$ be a neighborhood of $p$ contained in $W$. For each $\alpha, 1 \leqslant \alpha<\omega_{1}^{\beta<\alpha}$, let $U_{a}$ $=\bigcup_{p \in W \cap B_{a}} U_{p}$. Then $U_{1}, U_{2}, \ldots$ is an increasing, transfinite sequence of open subsets of $\boldsymbol{R}$-since $\bigcup_{1 \leqslant a<\omega_{1}} U_{\alpha}$ is Lindelöf, the sequence must, in reality, be countable. If we let $V_{\alpha}=\bigcup_{p \in W \cap B_{\alpha}} V_{p}$, it follows that, for some $\alpha_{0}<\omega_{1}, W=V_{1} \cup \ldots \cup V_{a_{0}}$. But $V_{\alpha}=U_{\alpha}-\left(\bigcup_{\beta<\alpha} P_{\beta}\right)$ is easily an $F_{a}$ in $\boldsymbol{R}$ and thus in $X$, so that $W=V_{1} \cup \ldots \cup V_{\alpha_{0}}$ is an $F_{a_{0}}$ in $X$.

Thus $X$ has the Borel properties we asked for: every closed set is a $G_{a}^{y}$ for some $\alpha$, but closed sets exist for arbitrarily high $\alpha$ which are nontrivial $G_{a}$ sets. 
It is clear that $X$ is Hausdorff, since $X$ consists of the real line with a topology finer than usual. However, $X$ cannot be regular, as the following argument shows.

Since $P_{1}$ is not closed in the usual line topology we can find a limit point $p$ of $P_{1}$ such that $p \in P$ for some $a>1$. Let $U$ be any open set in the $X$-topology containing $P_{1}$. Then for some open set $U_{0}$ in the usual line topology, $P_{1} \subset U_{0} \subset U$. Let $V_{0}$ be an open set in the usual line topology with $p \in V_{0}$. Then $U_{0} \cap V_{0} \neq \varnothing$, and we are done if we show $U_{0} \cap\left(V_{0}-\bigcup P_{\beta}\right)$ $\neq \emptyset$. But otherwise $U_{0} \cap V_{0}$ is a non-empty (usual) open set contained in $\bigcup P_{\beta}$, while by construction each $P_{\beta}$ is nowhere dense in the line. Then $\bigcup_{\beta<a}^{\beta<\alpha} P_{\beta}$ would be a set of first category with interior in the line. This contradiction establishes that $X$ cannot be regular.

3. In any space $X$, let $\mathfrak{B}(X)$ and $\mathcal{H}(X)$ (or, just $\mathcal{B}$ and $\mathcal{H}$, if $X$ is fixed) denote, respectively, the family of Borel sets and the smallest family of sets:

(1) containing the closed sets,

(2) closed under countable intersection,

(3) closed under countable disjoint union.

Our purpose in this section is to show the existence of non-perfectly normal spaces in which $\mathcal{B}$ and $\mathcal{H}$ coincide. It is convenient to denote countable disjoint union by $\bar{\sigma}$, so that the sets of $\mathscr{H}$ occur in a transfinite sequence: $F, F_{\bar{\sigma}}^{-}, F_{\bar{\sigma} \delta}^{-}, F_{\bar{\sigma} \bar{\sigma} \bar{\sigma}}^{-}, \ldots$

Theorem. $\mathcal{B}=\mathfrak{H}$ for the space $X_{2}$ of Section 1 (with $A_{2}$ taken to be the set of rationals $Q$ ).

Proof. For convenience, denote $X_{2}$ by $X, A_{2}$ by $Q$ and $X-A_{2}$ (the irrationals) by $P$.

First, it is a simple matter to show that, for $q \in Q, X-\{q\}$ is an $F_{\bar{\sigma}}$. Next, if $A$ and $B$ are closed subsets of $X$, with $A \subset B$, then $B-A$ is an $F_{\sigma \bar{\sigma} \bar{\sigma}}$. For

$$
B-A=[B \cap(-A \cap P)] \cup[B \cap(-A \cap Q)],
$$

where the union is disjoint. The second term is countable and thus an $F_{\bar{\sigma}}$. The first term can be handled by showing that, whenever $D \subset P, D$ is an $F_{\bar{\sigma} \delta}$. Now $\bar{D}-D$ is countable (consisting only of rationals) and

$$
D=\bigcap_{q \in \bar{D}-D} \bar{D}-\{q\}
$$

so it suffices to note that, since $\bar{D}-q=[X-\{q\}] \cap \bar{D}, \bar{D}-\{q\}$ is an $F_{\bar{\sigma}}$.
Now if $\bigcup_{i=1}^{\infty} A_{i}$ is an $F_{\sigma}$ in $X$, we may assume $A_{1} \subset A_{2} \subset \ldots$ Then, from the above,

$$
\bigcup_{i=1}^{\infty} A_{i}=\bigcup_{i=1}^{\infty}\left[A_{i}-A_{i-1}\right]
$$

is an $F_{\sigma \delta}^{-} \dot{\sigma}$.

Since every subset of $X$ is an $F_{\sigma \delta}$ (each $A \subset X_{2}$ can be represented as $(A \cap Q) \cup(A \cap P)$ where $A \cap Q$ is an $F_{\sigma}$ and $A \cap P$ is open. Since open subsets of $X_{2}$ are $F_{\sigma \delta}$ by Section $1, A$ is an $F_{\sigma \delta}$ every subset of $X$ is an $F_{\sigma \delta \delta \delta}$. This proves the theorem.

It is much easier to prove that, in the same space $X=X_{2}$, the family of Borel sets coincides with the smallest family

(1) containing the open sets,

(2) closed under countable intersection,

(3) closed under countable disjoint union.

\section{References}

[1] Z. Frolik, A contribution to the descriptive theory of sets, Czech. Acad. Sci., 1962, pp. 157-173.

[2] K. Kuratowski, Topologie, I, 4th ed., Monografie Matematyczne 20, Warszawa 1958.

[3] E. Michael, The product of a normal space and a metric space need not be normal, Bull. Amer. Math. Soc. 69 (1963), pp. 375-376.

Reçu par la Rédaction le 2.11. 1967 